\title{
RELATIONSHIPS BETWEEN CONTINUUM NEIGHBORHOODS \\ IN INVERSE LIMIT SPACES AND SEPARATIONS IN INVERSE LIMIT SEQUENCES
}

\author{
HARVEY S. DAVIS
}

\begin{abstract}
The main result of this paper is the following theorem. Let $\left\{X_{\alpha}, f_{\alpha \beta}, \alpha, \beta \in I\right\}$ be an inverse system of compact Hausdorff spaces and continuous onto maps with inverse limit $X$. Let $p \in X$ and $A$ be closed in $X$. There exists a continuum neighborhood of $p$ disjoint from $A$ if and only if there exists $\alpha \in I$ and disjoint sets $U$ and $V$ open in $X_{\alpha}$, neighborhoods respectively of $p_{\alpha}$ and $A_{\alpha}$ such that for all $\beta>\alpha, f_{\alpha \beta}^{-1}(U)$ lies in a single component of $X_{\beta}-f_{\alpha \beta}^{-1}(V)$. This is Theorem B of the text.
\end{abstract}

Introduction. Throughout this paper the conventions and elementary results on inverse limits of topological spaces are those given in Bourbaki [3] except when noted otherwise. $(I, \leqslant)$ is a directed set, $\left\{X_{\alpha} \mid \alpha \in I\right\}$ is a collection of nonvoid compact Hausdorff spaces and, for every pair $(\alpha, \beta)$ where $\alpha \leqslant \beta$, $f_{\alpha \beta}: X_{\beta} \rightarrow X_{\alpha}$ is continuous. The maps $f_{\alpha \beta}$ are not necessarily onto. $\left(X_{\alpha}, f_{\alpha \beta}\right)$ is an inverse system of topological spaces with inverse limit $X$ and canonical maps $f_{\alpha}: X \rightarrow X_{\alpha}$, for all $\alpha$. If $A \subseteq X, p \in X$ then denote $f_{\alpha}(A)=A_{\alpha}$ and $f_{\alpha}(p)=p_{\alpha}$.

If $A$ is any subset of a topological space $S$ then $\mathcal{T}(A)$ will denote the collection of all open (in $S$ ) subsets of $A$ and $\mathscr{F}(A)$ will denote the collection of all closed (in $S$ ) subsets of $A$. $\operatorname{Int}(A)$ will denote the interior of $A$ and $\mathrm{Clo}(A)$ will denote the closure of $A$.

$W$ is a subcontinuum of a topological space $S$ if and only if $W$ is compact, Hausdorff and connected. $W$ is a continuum neighborhood of a point $p$ of $S$ if and only if $p$ is an element of the interior of $W$. If $A$ is any subset of $S$ then $T(A)$ will denote the set of those points for which there does not exist a continuum neighborhood disjoint from $A$.

Let $\alpha \in I .\left\{W_{\beta} \mid \beta \geqslant \alpha\right\}$ denotes an inverse system of subcontinua if and only if for all $\beta \geqslant \alpha, W_{\beta}$ is a subcontinuum of $X_{\beta}$, and $\left(W_{\beta}, f_{\alpha \beta} \mid W_{\beta}\right)$ is an inverse system.

Let $S$ be a compact Hausdorff space. The following dictionary provides translations of some common properties of $S$ into properties of the set function $T$ defined on $\{A \mid A \subseteq S\}$.

Received by the editors September 29, 1975 and, in revised form, July 26, 1976.

AMS (MOS) subject classifications (1970). Primary 54F15; Secondary 54B25.

Key words and phrases. Continuum neighborhood, set function $T$, inverse limit, compact Hausdorff space. 
$S$ is connected im kleinem at $p \in S$ if and only if for every $A \in \mathscr{F}(S)$, if $p \notin A$ then $p \notin T(A)$.

$S$ is semi-locally connected at $p \in S$ if and only if $T(p)=p$.

$S$ is aposyndetic at $q$ with respect to $p$ if and only if $q \notin T(p)$.

$S$ is an indecomposable continuum if and only if for every $p \in S, T(p)=S$.

Other material on the set function $T$ can be found in [1], [2], [4], [5].

A. Fundamental theorems. Theorems A1 and A2 establish necessary and sufficient conditions on the inverse limit sequence that a given point $p$ is not an element of $T(A)$ for a given closed set $A$.

Lemma A1. Let $p \in X$ and $A \in \mathscr{F}(X)$. If there exists $\alpha \in I, U \in \mathcal{T}\left(X_{\alpha}\right)$, $V \in \mathcal{T}\left(X_{\alpha}\right)$ and an inverse system of subcontinua $\left\{W_{\beta} \mid \beta \geqslant \alpha\right\}$ such that $p_{\alpha} \in U, A_{\alpha} \subseteq V$ and such that for all $\beta \geqslant \alpha, f_{\alpha \beta}^{-1}(U) \subseteq W_{\beta} \subseteq X_{\beta}-f_{\alpha \beta}^{-1}(V)$, then $p \notin T(A)$.

Proof. Let $W$ be the canonical image of inv $\lim W_{\beta}$ in $X$. Since each $W_{\beta}$ is a continuum, $W$ is a continuum. Since, for each $\beta \geqslant \alpha, f_{\alpha}^{-1}(U) \subseteq$ $\operatorname{Clo}\left(f_{\alpha}^{-1}(U)\right) \subseteq \operatorname{Clo}\left(f_{\beta}^{-1} f_{\alpha \beta}^{-1}(U)\right) \subseteq f_{\beta}^{-1}\left(W_{\beta}\right)$ and since $W=\cap\left\{f_{\beta}^{-1}\left(W_{\beta}\right) \mid \beta\right.$ $\geqslant \alpha\}, f_{\alpha}^{-1}(U) \subseteq W$. Since $p \in f_{\alpha}^{-1}(U), p \in \operatorname{Int}(W)$. Since, for all $\beta \geqslant \alpha$,

$$
f_{\beta}^{-1}\left(W_{\beta}\right) \subseteq X-f_{\beta}^{-1} f_{\alpha \beta}^{-1}(V)=X-f_{\alpha}^{-1}(V)=f_{\alpha}^{-1}\left(X_{\alpha}-V\right) \subseteq X-A,
$$

$\cap\left\{f_{\beta}^{-1}\left(W_{\beta}\right) \mid \beta \geqslant \alpha\right\} \subseteq X-A$, so $W \cap A=\varnothing$. Therefore $p \notin T(A)$.

Lemma A2. Let $p \in X$ and $A \in \mathscr{F}(X)$. If $p \notin T(A)$ then there exists $\alpha \in I$, $U \in \mathcal{T}\left(X_{\alpha}\right), V \in \mathcal{T}\left(X_{\alpha}\right)$ and an inverse system of subcontinua $\left\{W_{\beta} \mid \beta \geqslant \alpha\right\}$ such that $p_{\alpha} \in U, A_{\alpha} \subseteq V$ and for all $\beta \geqslant \alpha$,

$$
f_{\alpha \beta}^{-1}(U) \cap f_{\beta}(X) \subseteq W_{\beta} \subseteq f_{\beta}(X)-f_{\alpha \beta}^{-1}(V) .
$$

Proof. Since $p \notin T(A)$, there exists a subcontinuum $W$ of $X$ such that $p \in \operatorname{Int}(W)$ and $W \cap A=\varnothing$. There exists $\alpha(p) \in I, U(p) \in \mathcal{T}\left(X_{\alpha(p)}\right)$ such that $p \in f_{\alpha(p)}^{-1}(U(p)) \subseteq \operatorname{Int}(W)$ since $\left\{f_{\alpha}^{-1}(U) \mid \alpha \in I, U \in \mathcal{T}\left(X_{\alpha}\right)\right\}$ is a basis for $\mathcal{T}(X)$. Similarly, for each $x \in A$, there exists $\alpha(x) \in I, U(x) \in \mathcal{T}\left(X_{\alpha(x)}\right)$ such that $x \in f_{\alpha(x)}^{-1}(U(x)) \subseteq X-W$. Let $\left\{f_{\alpha(i)}^{-1}\left(U_{i}\right) \mid i=1, \ldots, n\right\}$ be a finite subcover of the open cover $\left\{f_{\alpha(x)}^{-1}(U(x)) \mid x \in A\right\}$ of the compact set $A$. Since $I$ is a directed set, there exists $\alpha \in I$ such that $\alpha \geqslant \alpha(p)$ and for $i=1, \ldots, n$, $\alpha \geqslant \alpha(i)$. Let $U=f_{\alpha(p) \alpha}^{-1}(U(p))$ and $V=f_{\alpha(1) \alpha}^{-1}\left(U_{1}\right) \cup \cdots \cup f_{\alpha(n) \alpha}^{-1}\left(U_{n}\right)$. For $\beta \geqslant \alpha$ let $W_{\beta}=f_{\beta}(W)$.

Let $\beta \geqslant \alpha$ and let $z \in f_{\alpha \beta}^{-1}(U) \cap f_{\beta}(X)$. Since $z \in f_{\beta}(X)$, there exists $x \in X$ such that $x_{\beta}=z$. Since $f_{\alpha(p) \beta}(z) \in U(p), x_{\alpha(p)} \in U(p)$. Therefore $x \in f_{\alpha(p)}^{-1}(U(p)) \subseteq \operatorname{Int}(W) \subseteq W$. Thus $z=x_{\beta} \in f_{\beta}(W)=W_{\beta}$. So $f_{\alpha \beta}^{-1}(U) \cap$ $f_{\beta}(X) \subseteq W_{\beta}$. Now let $z \in W_{\beta}=f_{\beta}(W)$. Let $z=x_{\beta}$ for some $x \in W$. Clearly $z \in f_{\beta}(X)$. Suppose $z \in f_{\alpha \beta}^{-1}(V)$. There exists $i \leqslant n$ such that $f_{\alpha(i) \beta}(z) \in U_{i}$ so $x_{\alpha(i)} \in U_{i}$. Therefore $x \notin W$. This contradiction establishes that $W_{\beta} \subseteq f_{\beta}(X)$ $-f_{\alpha \beta}^{-1}(V)$. 
TheOREM A1. Let $p \in X$ and $A \in \mathscr{F}(X)$. The following are equivalent.

(a) $p \notin T(A)$.

(b) There exists $\alpha \in I, U \in \mathcal{T}\left(X_{\alpha}\right), V \in \mathcal{T}\left(X_{\alpha}\right)$ and an inverse system of subcontinua $\left\{W_{\beta} \mid \beta \geqslant \alpha\right\}$ such that $p_{\alpha} \in U, A_{\alpha} \subseteq V$ and for all $\beta \geqslant \alpha$,

$$
f_{\alpha \beta}^{-1}(U) \cap f_{\beta}(X) \subseteq W_{\beta} \subseteq f_{\beta}(X)-f_{\alpha \beta}^{-1}(V) \text {. }
$$

Proof. This follows directly from Lemmas A1 and A2 once it is noted that if $W$ is the canonical image of inv $\lim W_{\beta}$ in $X$ then for all $\beta, f_{\beta}(W)=f_{\beta}(X)$ $\cap W_{\beta}, f_{\beta}(W)$ is a continuum and $W$ is the canonical image of inv $\lim f_{\beta}(W)$. The relations in Lemma Al imply that $f_{\alpha \beta}^{-1}(U) \cap f_{\beta}(X) \subseteq f_{\beta}(W) \subseteq f_{\beta}(X)-$ $f_{\alpha \beta}^{-1}(V)$.

Theorem A2. Suppose that each $f_{\alpha \beta}$ is onto. Let $p \in X$ and $A \in \mathscr{F}(X)$. The following are equivalent.

(a) $p \notin T(A)$.

(b) There exists $\alpha \in I, U \in \mathcal{T}\left(X_{\alpha}\right), V \in \mathcal{T}\left(X_{\alpha}\right)$ and an inverse system of subcontinua $\left\{W_{\beta} \mid \beta \geqslant \alpha\right\}$ such that $p_{\alpha} \in U, A_{\alpha} \subseteq V$ and such that for all $\beta \geqslant \alpha$,

$$
f_{\alpha \beta}^{-1}(U) \subseteq W_{\beta} \subseteq X_{\beta}-f_{\alpha \beta}^{-1}(V)
$$

Proof. This follows directly from Theorem Al once it is noted that, since each $X_{\alpha}$ is compact, the hypothesis that each $f_{\alpha \beta}$ is onto forces each $f_{\alpha}$ to be onto.

Corollary A1. Suppose that each $f_{\alpha \beta}$ is onto. Let $J$ be a cofinal subset of $I$ and let $A \in \mathcal{F}(X)$. Then

$$
\cap\left\{f_{\alpha}^{-1}\left(T\left(A_{\alpha}\right)\right) \mid \alpha \in J\right\} \subseteq T(A) .
$$

Proof. Suppose $p \in X-T(A)$. Let $\alpha, U, V$ and $\left\{W_{\beta}\right\}$ be as in Theorem A2. Since $J$ is cofinal, there exists $\beta \in J$ such that $\beta \geqslant \alpha$. But then $p_{\beta} \in$ $f_{\alpha \beta}^{-1}(U) \subseteq W_{\beta} \subseteq X_{\beta}-f_{\alpha \beta}^{-1}(V) \subseteq X_{\beta}-A_{\beta}$. Therefore $p_{\beta} \notin T\left(A_{\beta}\right)$ so $p \notin$ $f_{\beta}^{-1}\left(T\left(A_{\beta}\right)\right)$. Thus $p \notin \cap\left\{f_{\gamma}^{-1}\left(T\left(A_{\gamma}\right)\right) \mid \gamma \in J\right\}$.

COROLlary A2. Suppose that each $f_{\alpha \beta}$ is onto. Let $J$ be a cofinal subset of $I$ and let $A \in \mathscr{F}(X)$. If for all $\gamma, \delta \in I$ where $\gamma \leqslant \delta, f_{\gamma \delta}$ is monotone, then

$$
T(A)=\cap\left\{f_{\gamma}^{-1}\left(T\left(A_{\gamma}\right)\right) \mid \gamma \in J\right\} \text {. }
$$

Proof. Suppose $p \in X-\cap\left\{f_{\gamma}^{-1}\left(T\left(A_{\gamma}\right)\right) \mid \gamma \in J\right\}$. There exists $\alpha$ such that $p_{\alpha} \notin T\left(A_{\alpha}\right)$ so there exist $U, V \in \mathcal{T}\left(X_{\alpha}\right)$ and a continuum $W$ such that $p_{\alpha} \in U \subseteq W \subseteq X_{\alpha}-V \subseteq X_{\alpha}-A_{\alpha}$. Now let $\beta \geqslant \alpha$ and let $W_{\beta}=f_{\alpha \beta}^{-1}(W)$. Since $f_{\alpha \beta}$ is monotone, $W_{\beta}$ is a continuum. It is clear that $\left\{W_{\beta} \mid \beta \geqslant \alpha\right\}$ is an inverse system of subcontinua and also that $f_{\alpha \beta}^{-1}(U) \subseteq W_{\beta} \subseteq X_{\beta}-f_{\alpha \beta}^{-1}(V)$. Hence, by Theorem A2, $p \notin T(A)$ so $T(A) \subseteq \bigcap\left\{f_{\gamma}^{-1}\left(T\left(A_{\gamma}\right)\right) \mid \gamma \in J\right\}$. Com- 
bining this with the above corollary gives the equality.

\section{B. Separations in inverse limit sequences.}

Definition. Let $M$ and $N$ be subsets of a topological space $S$. $M$ separates $N$ in $S$ if and only if there exist sets $P$ and $Q$ such that $S-M=P \cup Q$, $P \cap N \neq \varnothing \neq Q \cap N$ and $(P \cap \operatorname{Clo}(Q)) \cup(Q \cap \operatorname{Clo}(P))=\varnothing$.

Remarks. If $N \subseteq M$ then $M$ does not separate $N$ in $S$. If $M$ does not separate $N$ in $S$ and $L \subseteq M$ then $L$ does not separate $N$ in $S$.

Definitions. Let $A \in \mathcal{F}(X)$ and $p \in X . p \notin S(A)$ if and only if there exists $\alpha \in I, U \in \mathcal{T}\left(X_{\alpha}\right)$ and $v \in \mathcal{T}\left(X_{\alpha}\right)$ such that $p_{\alpha} \in U, A_{\alpha} \subseteq V, U \cap V$ $=\varnothing$, and, for all $\beta \geqslant \alpha, f_{\alpha \beta}^{-1}(V)$ does not separate $f_{\alpha \beta}^{-1}(U)$ in $X_{\beta}$.

RemarK. Let $A \in \mathscr{F}(X)$. It is immediate from the definitions that $A \subseteq$ $S(A)$.

TheOrem Bl. Let $A \in \mathscr{F}(X) . T(A) \subseteq S(A)$.

Proof. Suppose $p \notin S(A)$. Let $\alpha, U, V$ be as in the above definition of $S$. Now let $\beta \geqslant \alpha$. Since $X_{\beta}$ is compact Hausdorff and $f_{\alpha \beta}^{-1}(V)$ does not separate $f_{\alpha \beta}^{-1}(U)$ in $X_{\beta}, f_{\alpha \beta}^{-1}(U)$ lies entirely in a single component $W_{\beta}$ of $X_{\beta}$ $f_{\alpha \beta}^{-1}(V)$. It is clear that $W_{\beta}$ is a subcontinuum of $X_{\beta}$, that $f_{\alpha \beta}^{-1}(U) \subseteq W_{\beta}$ and that $W_{\beta} \subseteq X_{\beta}-f_{\alpha \beta}^{-1}(V)$. For all $\beta \geqslant \alpha$, choose such a $W_{\beta}$. Now let $\gamma \geqslant \beta$ $\geqslant \alpha$. Note that $f_{\beta \gamma}\left(W_{\gamma}\right) \subseteq f_{\beta \gamma}\left(X_{\gamma}-f_{\alpha \gamma}^{-1}(V)\right) \subseteq X_{\beta}-f_{\alpha \beta}^{-1}(V)$. Also note that $f_{\alpha \beta}^{-1}(U)=f_{\beta \gamma}\left(f_{\alpha \gamma}^{-1}(U)\right) \subseteq f_{\beta \gamma}\left(W_{\gamma}\right)$ so that $f_{\alpha \beta}^{-1}(U) \subseteq f_{\beta \gamma}\left(W_{\gamma}\right) \cap W_{\beta}$. Since $W_{\gamma}$ is connected and $f_{\beta \gamma}\left(W_{\gamma}\right) \cap W_{\beta} \neq \varnothing, f_{\beta \gamma}\left(W_{\gamma}\right) \subseteq W_{\beta}$ so $\left\{W_{\beta}\left|f_{\beta \gamma}\right|_{w_{\beta}}\right\}$ is an inverse of subcontinua. Therefore by Lemma Al, $p \notin T(A)$. Thus $T(A) \subseteq$ $S(A)$.

TheOREM B2. Suppose that each $f_{\alpha \beta}$ is onto. Let $A \in \mathscr{F}(X) . S(A)=T(A)$.

Proof. Suppose $p \notin T(A)$. Let $\alpha, U, V$ and $\left\{W_{\beta}\right\}$ be as in Theorem A2. Note that $A_{\alpha} \subseteq V, p_{\alpha} \in U$ and that $U \cap V=\varnothing$. Now let $\beta \geqslant \alpha$. Since $f_{\alpha \beta}^{-1}(U) \subseteq W_{\beta}$ and $W_{\beta} \cap f_{\alpha \beta}^{-1}(V)=\varnothing$, it is clear that $f_{\alpha \beta}^{-1}(V)$ does not separate $f_{\alpha \beta}^{-1}(U)$ in $X_{\beta}$. Hence $p \notin S(A)$ and thus $S(A) \subseteq T(A)$. By Theorem $\mathrm{B} 1, S(A)=T(A)$.

The main result can now be stated.

TheOREM B. Suppose that each $f_{\alpha \beta}$ is onto. Let $A \in \mathscr{F}(X)$, and $p \in X$. There exists a continuum neighborhood of $p$ disjoint from $A$ if and only if there exists $\alpha \in I$ and disjoint sets $U$ and $V$ open in $X_{\alpha}$, neighborhoods respectively of $p_{\alpha}$ and $A_{\alpha}$ such that for all $\beta \geqslant \alpha, f_{\alpha \beta}^{-1}(U)$ lies in a single component of $X_{\beta}-$ $f_{\alpha \beta}^{-1}(V)$.

Proof. Once it is noted that in a compact Hausdorff space the failure of an open set $G$ to separate an open set $D$ is equivalent to the existence of a component $K$ of $S-G$ that contains $D$ the theorem follows as a corollary to Theorem B2. 


\section{BIBLIOGRAPHY}

1. D. P. Bellamy and H. S. Davis, Continuum neighborhoods and filterbases, Proc. Amer. Math. Soc. 27 (1971), 371-374. MR 43 \#2653.

2. D. E. Bennett, A characterization of locally connectedness by means of the set function $T$, Fund. Math. 86 (1974), 137-141. MR 50 \# 11189.

3. N. Bourbaki, General topology. Parts 1 and 2, Addison-Wesley, Reading, Mass., 1966. MR 34 \# 5044a, 5044b.

4. H. S. Davis, D. P. Stadtlander and P. M. Swingle, Properties of the set function $T^{n}$, Portugal. Math. 21 (1962), 113-133. MR 25 \#5501.

5. H. S. Davis, A note on connectedness im kleinen, Proc. Amer. Math. Soc. 19 (1968), 1237-1241. MR 40 \#8021.

Department of Mathematics, Michigan State University, East Lansing, Michigan 48824 